\title{
Novel Neurospora crassa mutants with altered synthesis of polyunsaturated fatty acids
}

\author{
Marta Goodrich-Tanrikulu, Jiann-Tsyh Lin, Allan E. Stafford, \\ Martina I. Makapugay, Thomas A. McKeon and Glenn Fuller
}

Author for correspondence: Marta Goodrich-Tanrikulu. Tel: +1 510559 5627. Fax : +1 5105595777.

United States Department of Agriculture, Agricultural Research Service, Western Regional Research Center, 800 Buchanan Street, Albany, CA 94710, USA

\begin{abstract}
Five new mutants of Neurospora crassa that require supplementation with unsaturated fatty acids have been isolated. The mutants, designated pfa, are impaired in the synthesis of the polyunsaturated fatty acids $\alpha$-linoleic or $\alpha$-linolenic acid, but are able to synthesize oleic acid. The pfa mutants are thus distinct from previously described ufa mutants, which are unable to synthesize oleic acid. The five pfa mutants map to distinct loci, and have characteristic patterns of incorporation of $\left[{ }^{14} \mathrm{C}\right]$ acetate and $\left[{ }^{14} \mathrm{C}\right]$ oleate into their fatty acids.
\end{abstract}

Keywords: Neurospora crassa, fatty acid desaturation, lipids, polyunsaturates

\section{INTRODUCTION}

Polyunsaturated fatty acids (FA) are ubiquitous components of membrane lipids and are often components of storage lipids in eukaryotic cells. They are important determinants of lipid physical properties, such as membrane fluidity and the melting temperatures of triglyceride-based oils. The levels of polyunsaturates in membrane lipids usually increase in response to cold treatment, conferring greater membrane fluidity, and are believed to be an important factor in temperature adaptation (Weete, 1980; Martin et al., 1981). The polyunsaturates $\alpha$-linoleate $\left(18: 2 \Delta^{9,12}\right)$ and $\alpha$-linolenate $\left(18: 3 \Delta^{9,12,15}\right)$ are also essential dietary components for human nutrition. The major source of these FA in the human diet is from plant-derived oils.

Aside from plants, few other organisms are able to complete the de novo pathway of $\alpha-18: 3$ biosynthesis. Among these organisms are some fungi (Weete, 1980). The biosynthetic pathway for polyunsaturated FA is thought to be similar for higher plants and fungi. Plants contain at least two routes for the synthesis of polyunsaturates (one localized in the chloroplast, one in microsomes, probably ER), and the ER route is apparently the major one for the synthesis of polyunsaturatecontaining TG (Frentzen, 1993). In fungi, the synthesis of polyunsaturates is believed to occur in the ER (Weete, 1980). For fungi and the ER-localized pathway in plants,

Abbreviations: 16:0, palmitic acid; 18:0, stearic acid; 18:1, oleic acid; 18:2, linoleic acid; 18:3, linolenic acid; DG, diacylglycerol; ER, endoplasmic reticulum; FA, fatty acid; G-3-P, glycerol 3-phosphate; LPA, lysophosphatidic acid; PA, phosphatidic acid; PC, phosphatidylcholine; PE, phosphatidylethanolamine; PS, phosphatidylserine; TG, triacylglycerol. desaturation of the immediate precursor 18:1 occurs while it is esterified to the phospholipid PC (Weete, 1980; Heinz, 1993).

Mutants in the pathway of FA desaturation would be useful for a better understanding of the regulation of these pathways and for gene isolation. In $\alpha-18: 3$-producing fungi, comparatively little information is available on the desaturation pathway. The filamentous fungus Neurospora crassa synthesizes $\alpha-18: 3$, and is amenable to classical and molecular genetic techniques. Additionally, some biochemical information on the pathway of desaturation in Neurospora is available, and mutants for the desaturation of 18:0 to 18:1 (ufa) (Scott, 1977a; Goodrich-Tanrikulu et al., 1994) and for the biosynthesis of PC (chol-1, chol-2) (Horowitz \& Beadle, 1943; Horowitz et al., 1945; Scarborough \& Nyc, 1967) have been isolated. Mutants defective in the desaturation of 18:1 or $\alpha-18: 2$ have not been described, aside from reports that mutants defective in the synthesis of NADPH have reduced levels of $\alpha-18: 3$ (Brody \& Nyc, 1970; Nyc \& Brody, 1971), and that ufa mutants may also be impaired in subsequent desaturation steps (Scott, 1977b; Gabrielides et al., 1982; GoodrichTanrikulu et al., 1994).

Mutants in the biosynthesis of polyunsaturated FA have been isolated based on impaired growth at low temperatures from the cyanobacterium Synechocystis PCC 6803 (Wada \& Murata, 1989), which utilizes a chloroplast-like pathway to synthesize $\alpha-18: 2$, and from the fungus Mortierella, which synthesizes $\gamma$-linolenate $\left(18: 3 \Delta^{6,9,12}\right)$ and arachidonate $\left(20: 4 \Delta^{5,8,11,14}\right)$ (Jareonkitmongkol et al., 1992). Using an $\alpha-18: 3$ requirement for growth at low temperatures, we have isolated several Neurospora mutants with altered synthesis of unsaturated FA. We have previously described two 
mutants which synthesize only saturated FA (GoodrichTanrikulu et al., 1994). Here, we describe FA composition, synthesis, and desaturation by mutants with defects in the synthesis of polyunsaturated FA.

\section{METHODS}

Cultures, media and crosses. The wild-type Neurospora crassa strains 74-OR23-1A (wt74A) and 74-OR8-1a (wt74a), and the strain alcoy; csp-2 were obtained from the Fungal Genetics Stock Center, Department of Microbiology, University of Kansas Medical Center, Kansas City, KS, USA. The $a^{m 1} a d-3 B c y b-1$ strain was a gift of David D. Perkins, Stanford University.

Cultures were grown as described by Goodrich-Tanrikulu et al. (1994); unless otherwise noted, FA supplementation for all media was $1 \mathrm{mM} \alpha-18: 3$ (87\%, remainder $\alpha-18: 2$, prepared as described by Goodrich-Tanrikulu et al., 1994), solubilized with $1 \%(\mathrm{v} / \mathrm{v})$ of the detergent Tergitol NP40.

Preliminary genetic mapping was with the linkage tester strain alcoy;csp-2 as described by Goodrich-Tanrikulu et al. (1994), except the crossing medium was supplemented with $1 \%$ Tergitol NP40 and $5 \mathrm{mM}$ 18:3. Lines of interest which carried additional morphological mutations (three of five mutants) were backcrossed to wt74a to segregate out these characters.

Mutagenesis, selection and characterization of polyunsaturated FA mutants. Conidia of wt74A, suspended in tubes of sterile water at a density of $2 \times 10^{7} \mathrm{ml}^{-1}$, were mutagenized by exposure to $\gamma$-irradiation $\left({ }^{60} \mathrm{Co}\right.$ source) for $4 \mathrm{~h}$ at $100 \mathrm{~Gy} \mathrm{~h}^{-1}$, or by exposure to NTG or ICR-170 as described in Davis \& deSerres (1970). Mutant enrichment and preliminary screening were at $15^{\circ} \mathrm{C}$ in Vogel's minimal medium (Davis \& deSerres, 1970) as described by Goodrich-Tanrikulu et al. (1994). Lines which reproducibly grew faster with 18:3 supplementation (two additional transfers) were considered potential mutants in the synthesis of unsaturated FA, and were further screened to identify total FA biosynthetic profiles by radiolabelling with $\left[{ }^{14} \mathrm{C}\right]$ acetate at $15^{\circ} \mathrm{C}$ as described by Goodrich-Tanrikulu et al. (1994). In more detailed studies, liquid-grown cultures were radiolabelled with either $370 \mathrm{kBq}$ $\left[{ }^{14} \mathrm{C}\right]$ acetate (sodium salt, ICN, $1.48-2 \cdot 22 \mathrm{GBq} \mathrm{mmol}^{-1}$ ) or $37 \mathrm{kBq}\left[{ }^{14} \mathrm{C}\right]$ oleate (ICN, $1 \cdot 11-2 \cdot 22 \mathrm{GBq} \mathrm{mmol}^{-1}$ ) at $23{ }^{\circ} \mathrm{C}$. Lipids were extracted and analysed as described by GoodrichTanrikulu et al. (1994).

\section{RESULTS AND DISCUSSION}

\section{Mutant selection, genetic mapping, and growth characteristics}

Following mutagenesis and filtration enrichment, 26200 colonies (6000 from NTG, 2800 from ICR-170, and 17400 from $\gamma$-irradiation treatments, respectively) were screened for growth in the presence and absence of supplemental 18:3. In the screening of ICR-170- and NTG-treated conidia, several hundred lines showed improved growth through the preliminary screen and an additional transfer, but failed to grow reproducibly upon subsequent transfers. We suspected that some promising lines were being lost to reversion, and attempted to obtain nonreverting lines using $\gamma$-irradiation. A similar proportion of the $\gamma$-irradiated lines were 'lost' in a similar manner.

Of the total colonies screened, 44 showed a requirement for unsaturated FA supplementation through two ad- ditional transfers at $15^{\circ} \mathrm{C}$, and were further screened for ability to synthesize $\mathrm{FA}$ de novo from $\left[{ }^{14} \mathrm{C}\right]$ acetate. Based on this assay, 15 lines showed reduced synthesis of polyunsaturated FA. The levels of labelled polyunsaturated FA remained within the wild-type range for all but five of the lines assayed. We have selected the designation $p f a$ for these five mutants. Four of the $p f a$ mutants synthesized no $18: 3$ at $15^{\circ} \mathrm{C}$; all but one of these $(p f a-1)$ also had reduced 18:2 (Table 1). The remaining mutant $(p f a-5)$ synthesized only $40 \%$ of the $18: 3$ and $55 \%$ of the $18: 2$ of wild-type. Also at $23{ }^{\circ} \mathrm{C}$, all five mutants showed impaired synthesis of polyunsaturated FA (detailed below).

The five mutations were independent as shown by mapping and tests for allelism (Table 2). After backcrossing, the morphology of all of the mutants was wildtype. The mutants required unsaturated $\mathrm{FA}$ supplementation at $15^{\circ} \mathrm{C}, 23{ }^{\circ} \mathrm{C}$, and $30^{\circ} \mathrm{C}$, except for the mutant $p f a-1$, which required supplementation at $15^{\circ} \mathrm{C}$, but grew very slowly (requiring several weeks to germinate) without supplementation at the higher temperatures. Germination of mutant conidiospores was delayed by several days (several weeks for $p f a-3$ ) compared to wildtype. Thereafter, the mutants grew at nearer wild-type rates. All of the mutants grew more slowly than wild-type despite supplementation, suggesting either that the level of supplementation was suboptimal or that FA supplementation only partially compensated for the mutation. A similar lack of complete restoration of wild-type phenotype by FA supplementation has been noted in the cel mutant of Neurospora (Coté \& Brody, 1987), which is defective in formation of 16:0.

18:3 appeared to be the optimal FA supplement for four of the $p f a$ mutants. The mutant $p f a-4$, however, unexpectedly grew more vigorously when supplemented with $1 \%$ (v/v) Tween 80 (which contains esterified 18:1) than with either $1-5 \mathrm{mM}$ free $18: 1(96 \%)$ or $18: 3(87 \%)$. The improved growth may be due to the higher level of available FA when supplied as a Tween ester.

Stocks of the strains could be maintained by cryopreservation as heterokaryons, but not reliably as homokaryons. Crosses were usually fertile when the mutants were sheltered as heterokaryons with the helper strain $a^{m 1}$ $a d-3 B c y b-1$, used as male parent, and supplemented with 18:3.

Each of the mutants was unstable, frequently reverting upon subculturing, as evidenced by regain of wild-type growth and FA biosynthetic profiles. Stocks of $p f a-1$ and $p f a-2$ also tended to become progressively impaired earlier in the desaturation pathway, either after subsequent transfers, or after backcrossing and selecting for FA supplementation requirement. This was evident from altered radiolabelling patterns with $\left[{ }^{14} \mathrm{C}\right]$ acetate; in extreme cases, the mutant patterns were identical to those of ufa mutants (Goodrich-Tanrikulu et al., 1994), which lack the ability to desaturate 18:0. The five $p f a$ mutants were non-allelic with ufa strains (Goodrich-Tanrikulu et al., 1994), as determined by heterokaryon tests. The accumulation of additional defects is reminiscent of some adenine and cysteine auxotrophs, which spontaneously 
Table 1. Results of screen for Neurospora mutants affecting the synthesis of polyunsaturated FA

Relative percentage incorporation of $\left[{ }^{14} \mathrm{C}\right]$ acetate $(37 \mathrm{kBq})$ into individual FA of total lipids after $24 \mathrm{~h}$ at $15^{\circ} \mathrm{C}$ is shown. Cultures were supplemented with $1 \mathrm{mM} 18: 3$. See Methods for further details of culture and labelling conditions. The range of wild-type values is expressed as mean incorporation $\pm \mathrm{SE}$ for three determinations. Total label incorporated ranged from approximately 0.3 to $1.7 \times 10^{6} \mathrm{c}$.p.m.

\begin{tabular}{|lcccccc|}
\hline \multirow{7}{*}{ Strain } & \multicolumn{7}{c|}{$\left[{ }^{14} \mathbf{C}\right]$ Acetate incorporation into: } \\
\cline { 2 - 7 } & $\mathbf{1 6 : 0}$ & $\mathbf{1 8 : 0}$ & $\mathbf{1 8 : 1}$ & $\mathbf{1 8 : 2}$ & $\mathbf{1 8 : 3}$ & Other* \\
\hline wt74A & $35 \cdot 0 \pm 5 \cdot 1$ & $9 \cdot 7 \pm 1 \cdot 3$ & $14 \cdot 3 \pm 0 \cdot 3$ & $32 \cdot 0 \pm 2 \cdot 1$ & $8 \cdot 0 \pm 3 \cdot 6$ & $1 \cdot 0 \pm 1 \cdot 0$ \\
$p f a-1$ & 47 & 4 & 17 & 31 & 0 & 0 \\
$p f a-2$ & 53 & 14 & 12 & 21 & 0 & 0 \\
$p f a-3$ & 48 & 15 & 15 & 21 & 0 & 0 \\
$p f a-4$ & 41 & 24 & 18 & 16 & 0 & 0 \\
$p f a-5$ & 36 & 23 & 18 & 18 & 3 & 1 \\
\hline
\end{tabular}

* Unidentified FA.

\section{Table 2. Map location of Neurospora pfa mutants}

Linkage is expressed as percentage of ascospores able to grow on unsupplemented medium that carry marker for given chromosome(s). Deviations from $50 \%$ (no linkage) are significant (Perkins, 1994). The mutations mapping to the same chromosomes were shown to be distinct by heterokaryon tests (Davis \& deSerres, 1970).

\begin{tabular}{|llcl|}
\hline Strain & Mutagen & Chromosome* & Linkage \\
\hline$p f a-1$ & $\gamma$-Irradiation & VII & $65 \%(53 / 81)$ \\
$p f a-2$ & $\gamma$-Irradiation & IV $/ \mathrm{V}$ & $68 \%(32 / 47)$ \\
$p f a-3$ & NTG & IV $/ \mathrm{V}$ & $71 \%(25 / 35)$ \\
$p f a-4$ & ICR-170 & VII & $67 \%(52 / 78)$ \\
$p f a-5$ & $\gamma$-Irradiation & I/II & $93 \%(40 / 43)$ \\
\hline
\end{tabular}

* Map location based on crosses to alcoy;csp-2.

accumulate secondary mutations in their respective biosynthetic pathways, suggesting a selective advantage for the double mutants (Mitchell \& Mitchell, 1950; Murray, 1965). The wild-type also tended to become impaired in $18: 2$ desaturation upon culturing in the presence of $18: 3$.

\section{FA synthesis and desaturation}

In Neurospora, $18: 1$ is most likely desaturated to $18: 2$ and 18:3 while esterified to PC (Baker \& Lynen, 1971; Weete, 1980). A mutation in one of the two desaturases would most directly reduce the level of polyunsaturated FA. However, because the mutations we identified were independent, more than two genes must affect the biosynthesis of polyunsaturated FA. Likely candidates are genes in the pathway for substrate synthesis and factors involved in desaturation, e.g., cytochrome $b_{5}$ and associated reductases. In Neurospora and other $\alpha-18: 3$ producing fungi, the major pathway for biosynthesis of phospholipids and triacylglycerols containing polyunsaturated FA is believed to be:

$\mathrm{G}-3-\mathrm{P} \rightarrow \mathrm{LPA} \rightarrow \mathrm{PA} \rightarrow \mathrm{PS} \rightarrow \mathrm{PE} \rightarrow \mathrm{PC} \rightarrow \mathrm{DG} \rightarrow \mathrm{TG}$,

with the acylation steps utilizing acyl CoA thioesters (Weete, 1980; Chopra \& Khuller, 1984; Baker \& Lynen, 1971). In addition, enrichment in the level of polyunsaturates probably occurs, as it does in plants (Frentzen, 1993; Heinz, 1993), via alternative, reversible reactions forming PC: the reaction of DG with CDP-choline, and acylation of lysoPC (Weete, 1980).

Prior studies have shown that mutations in pathway enzymes, or in synthesis of cofactors involved in the desaturations, can affect the levels of polyunsaturates: Neurospora mutants (chol-1, chol-2) blocked in the formation of PC by the major pathway of PE methylation differ from wild-type in FA composition and in the relative amount of $\mathrm{PC}$ and $\mathrm{PE}$, with the differences dependent on the level of choline supplementation (Hubbard \& Brody, 1975; Johnston et al., 1982). Also, mutants with reduced NADPH levels due to altered glucose-6-phosphate dehydrogenase produce only $20 \%$ of wild-type levels of 18:3 (Brody \& Nyc, 1970).

To obtain more information about where in the pathway the mutants we have identified might be affected, we examined the incorporation of polyunsaturated FA precursors into lipids of the mutants. First, we compared the incorporation of ${ }^{14} \mathrm{C}$ from acetate into individual phospholipids, glycolipids and neutral lipids of the mutants and wild-type under identical supplementation conditions. Incorporation patterns into lipids of each fraction were typically similar for the mutants and wildtype, and the majority of label was incorporated into TG, with one major exception: the mutant $p f a-3$ incorporated more label into free FA than did wild-type or other mutants. The relative amount of label incorporation into free FA of $p f a-3$ varied among experiments, ranging from about 25 to $100 \%$ of the label incorporated into TG. 


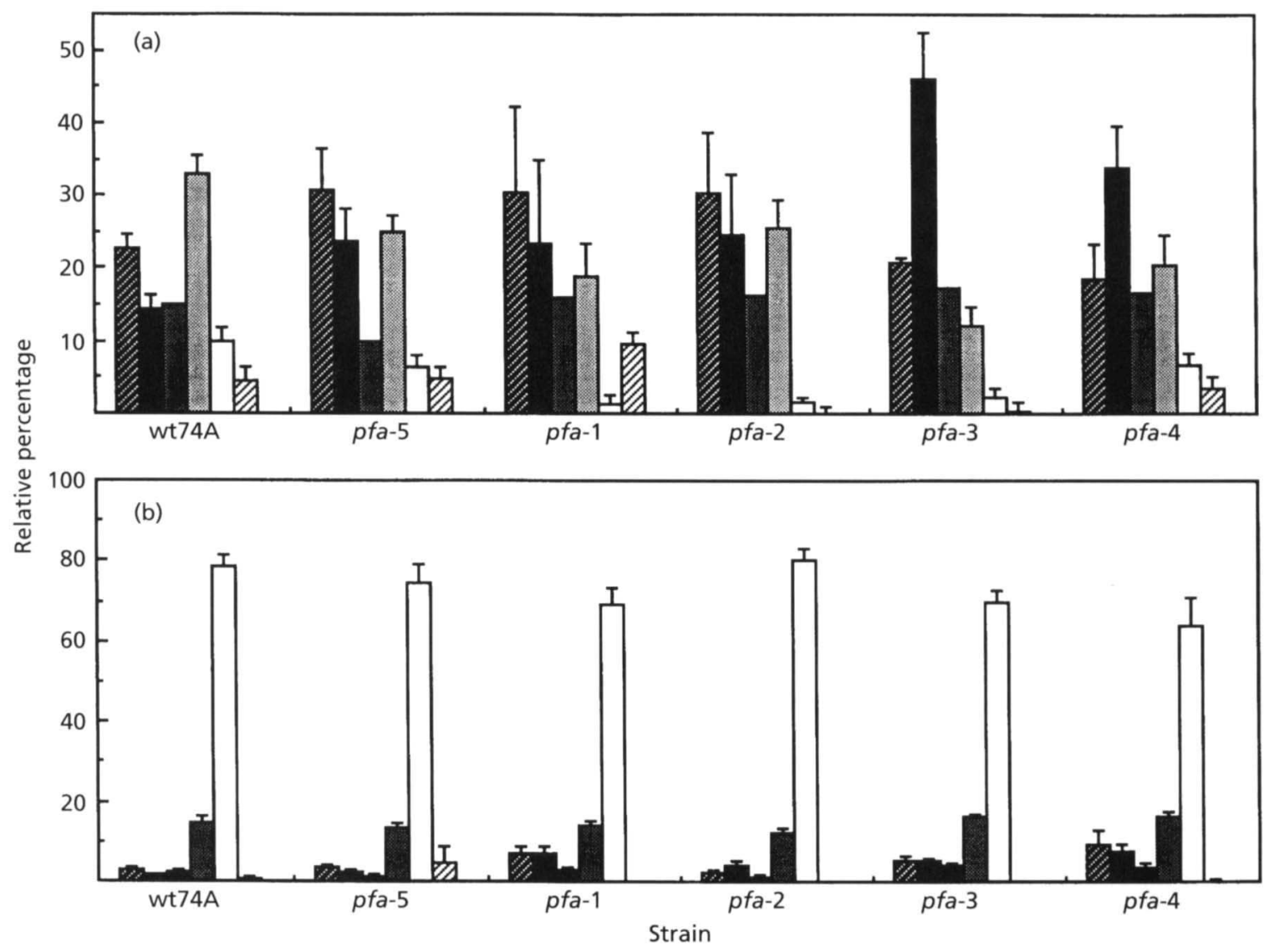

Fig. 1. TG synthesis by $18: 3$-supplemented Neurospora. (a) $\left[{ }^{14} \mathrm{C}\right]$ Acetate incorporation into TG; relative percentage incorporation into FA of TG $24 \mathrm{~h}$ after label addition to cultures grown at $23^{\circ} \mathrm{C}$. Cultures were radiolabelled and lipids extracted and separated as described in Methods and by Goodrich-Tanrikulu et al. (1994). FA methyl esters derived from the TG fraction were separated by HPLC, with radiodetection. (b) Relative percentage FA composition of TG by weight, based on GC analysis of methyl esters. Values are means of at least three determinations; error bars indicate SE. Absolute values for amount and ${ }^{14} \mathrm{C}$ incorporation per culture, approximately $1.5 \mathrm{mg} \mathrm{FA}$ and $1 \times 10^{7}$ c.p.m., respectively. Left to right: $\mathbf{B}, 16: 0 ; \square, 18: 0 ; \square, 18: 1 ;$, 18:2; $\square, 18: 3 ; \square$, other (unidentified).

$\left[{ }^{14} \mathrm{C}\right]$ Acetate incorporation into $\mathrm{FA}$ of $\mathrm{TG}$ showed distinctive differences for wild-type and mutant cultures (Fig. 1a). At $23^{\circ} \mathrm{C}$, the TG incorporation profiles differed somewhat from the total FA incorporation profiles used in screening at $15{ }^{\circ} \mathrm{C}$ (compare data of Table 1). At the lower temperature, more label remained in 16:0 for each of the strains, including two ( $p f a-3$ and $p f a-4)$ that accumulated label in 18:0 at higher temperatures (Fig. 1a). The higher proportion of label in 16:0 may be due to a slower rate of elongation and desaturation at the lower temperature. As observed previously (GoodrichTanrikulu et al., 1994), the FA composition of the TG was affected by the exogenous supplement, with the $18: 3$, and, to a lesser degree, 18:2 of the supplement being the predominant FA (Fig. 1b).

Since PC is believed to be the substrate for 18:1 and 18:2 desaturation, we compared the incorporation of $\left[{ }^{14} \mathrm{C}\right]$ acetate into FA of PC for each of the strains (Fig. 2). The amount of label incorporated into $18: 2$ was variable for the four mutants synthesizing the lowest levels of $18: 3$ de novo. The variable incorporation may result from mass- or age-dependent levels of 18:2 produced in Neurospora cultures (Coté \& Brody, 1987).

We also tested the ability of the mutants to desaturate exogenous 18:1 to polyunsaturated FA in vivo. At first inspection, the mutant phenotypes appeared to fall into three classes based on their ability to desaturate exogenous 18:1: $p f a-5$ closely resembled wild-type; $p f a-1$ and $p f a-2$ appeared to be impaired in the synthesis of $18: 3$, and were potentially defective in 18:2 desaturation; and $p f a-3$ and $p f a-4$ appeared to be impaired in the synthesis of both 18:2 and 18:3 (Fig. 3). However, in preliminary experiments, $p f a-4$ was able to convert the same proportion of labelled 18:1 to $18: 2$ in PC as wild-type (36\%). Thus, the defect in $p f a-4$ may be in $18: 2$ desaturation. For the $p f a-3$ mutant, the amount of ${ }^{14} \mathrm{C}$ from $18: 1$ incorporated into FA of PC was below our detection limit, so the distribution of label in PC is unknown. The patterns of incorporation of ${ }^{14} \mathrm{C}$ from $18: 1$ into neutral or phospholipids were unchanged from wild-type for any of the mutants (autoradiographs not shown), although for some of them, much of the label remained in the free FA 


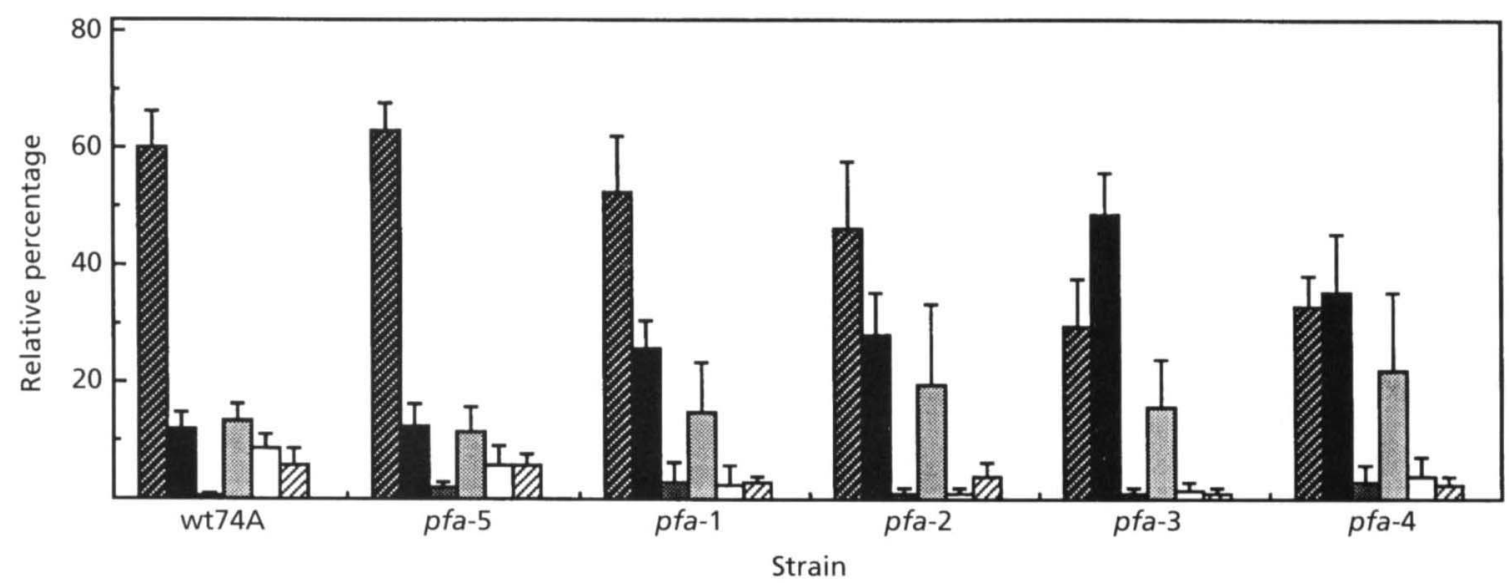

Fig. 2. $\left[{ }^{14} \mathrm{C}\right] \mathrm{Ace}$ tate incorporation into $\mathrm{PC}$ of 18:3-supplemented Neurospora. Details as for Fig. 1(a), except FA were derived from PC. (Absolute incorporation estimates can be obtained from Table 3.) Left to right: $\mathbf{0}, 16: 0 ; \mathbf{0}, 18: 0 ; \mathbf{}$, $18: 1 ;$, $18: 2 ; \square, 18: 3 ; \square$, other.

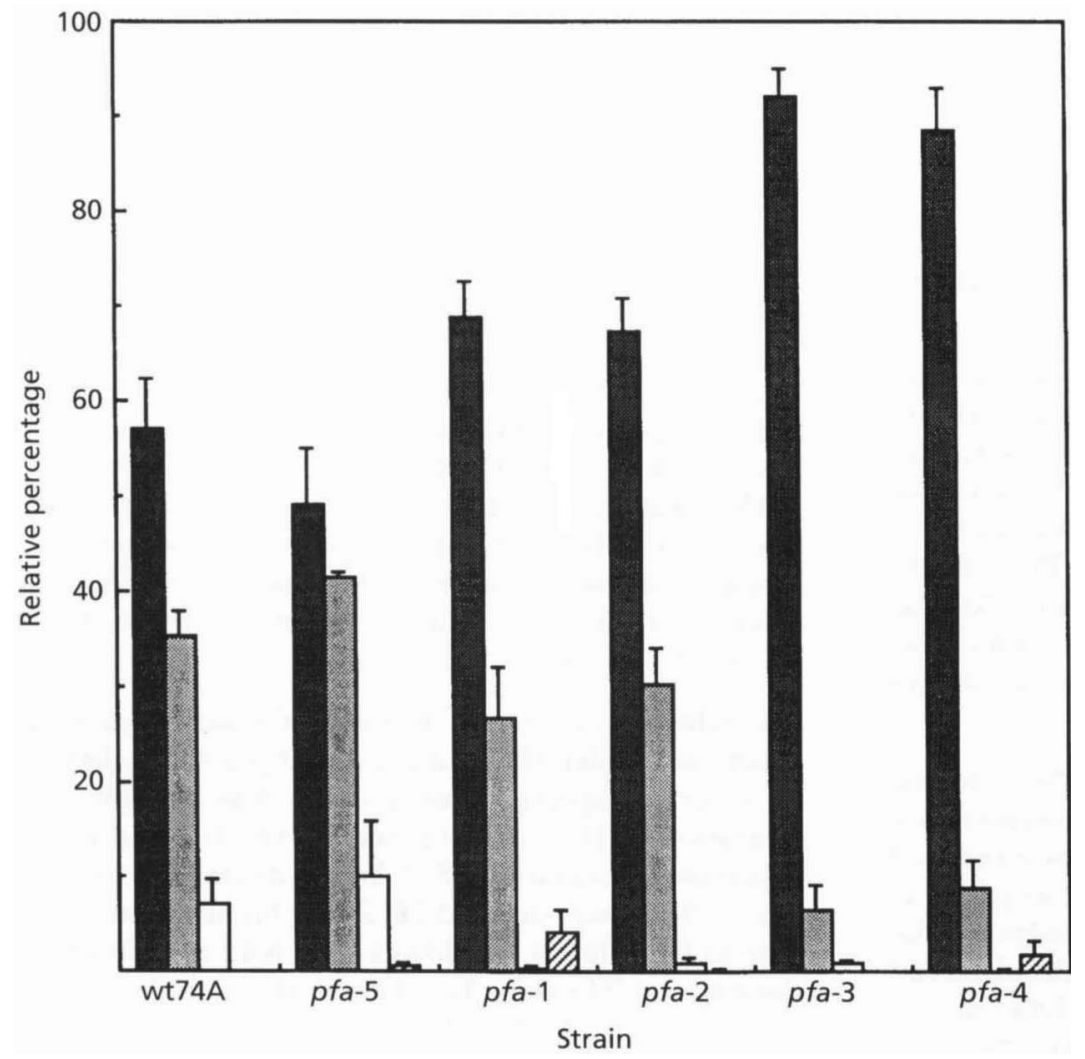

Fig. 3. $\left[{ }^{14} \mathrm{C}\right]$ Oleate incorporation into total FA of 18:3-supplemented Neurospora. Relative percentage incorporation into total FA of Neurospora cultures grown at $23^{\circ} \mathrm{C}$, $24 \mathrm{~h}$ after label addition, is shown. FA methyl esters were separated by HPLC, with radiodetection. Values are means of two (pfa-3, pfa-5) or three determinations; error bars indicate SE. Left to right: $\square, 18: 1 ;$, $18: 2 ; \square, 18: 3 ; \square$, other. band. This could have occurred due to impaired transfer of label into other lipids, or from unincorporated label in slow-growing cultures.

We also noted the presence of an unknown glycolipid which contained a small percentage of the total hydrolysable FA, predominantly as $18: 3$, in both wildtype and mutants (including the $u f a$ mutants previously described by Goodrich-Tanrikulu et al., 1994). It also incorporated labelled acetate (less than $2 \%$ of total label) at $24 \mathrm{~h}$ almost exclusively into $18: 3$, but did not incorporate exogenous $18: 1$. This lipid is perhaps involved in a minor pathway of FA desaturation.

\section{Probable basis of the mutations}

Several interpretations are possible for the data which we have gathered on these mutants. Below we describe further characteristic details of the individual mutants, 


\section{Table 3. Synthesis of major glycerolipids in Neurospora pfa mutants}

Cultures were labelled with $\left[{ }^{14} \mathrm{C}\right]$ acetate $(370 \mathrm{kBq})$ for $24 \mathrm{~h}$ at $23{ }^{\circ} \mathrm{C}$, and were supplemented with

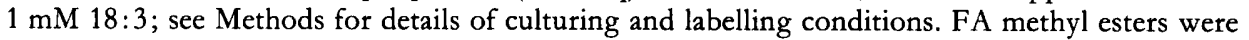
analysed following lipid class separation: amount (amt; relative weight percentage FA) was determined by $\mathrm{GC}$ with flame ionization detection, and $\left[{ }^{14} \mathrm{C}\right]$ acetate incorporation (incorp) was determined by HPLC with radiodetection. The absolute amount of material harvested varied between experiments, so relative values are more indicative of differences between strains. Average results of three or more sets of determinations (two for $p f a-2$ and $p f a-3$ DG incorporation) are shown.

\begin{tabular}{|c|c|c|c|c|c|c|c|c|}
\hline \multirow[t]{2}{*}{ Strain } & \multicolumn{2}{|c|}{$\begin{array}{c}\text { PE† } \\
\text { (\% TG) }\end{array}$} & \multicolumn{2}{|c|}{$\begin{array}{c}\text { PC† } \\
\text { (\% TG) }\end{array}$} & \multicolumn{2}{|c|}{$\begin{array}{c}\text { DG† } \\
(\% \mathrm{TG})\end{array}$} & \multicolumn{2}{|c|}{$\begin{array}{c}\text { TG } \ddagger \\
\text { (\%wt) }\end{array}$} \\
\hline & Amt & Incorp & Amt & Incorp & Amt & Incorp & Amt & Incorp \\
\hline wt74A & 4.5 & $10 \cdot 9$ & $3 \cdot 8$ & $12 \cdot 1$ & $0 \cdot 8$ & $2 \cdot 8$ & 100 & 100 \\
\hline$p f a-1$ & $6 \cdot 4$ & $10 \cdot 0$ & $8 \cdot 6$ & $15 \cdot 8$ & 3.7 & $4 \cdot 8$ & 27 & 64 \\
\hline$p f a-2$ & $4 \cdot 1$ & $9 \cdot 6$ & $6 \cdot 1$ & $12 \cdot 8$ & $1 \cdot 2$ & $2 \cdot 6$ & 81 & 70 \\
\hline$p f a-3$ & $3 \cdot 7$ & $12 \cdot 3$ & $6 \cdot 1$ & $9 \cdot 6$ & $3 \cdot 0$ & $3 \cdot 3$ & 43 & 82 \\
\hline$p f a-4$ & $3 \cdot 2$ & $15 \cdot 5$ & $8 \cdot 0$ & $22 \cdot 0^{*}$ & $5 \cdot 5$ & 3.5 & 10 & 19 \\
\hline$p f a-5$ & $7 \cdot 1$ & $13 \cdot 6$ & 4.9 & $15 \cdot 5$ & $2 \cdot 5$ & $6.9 *$ & 41 & 121 \\
\hline
\end{tabular}

* Relative values significantly different from wild-type ( $95 \%$, Student's $t$-test).

† Expressed as percentages relative to TG (normalized to $100 \%$ ).

$\ddagger$ Expressed relative to wild-type TG (absolute values for amount and ${ }^{14} \mathrm{C}$ incorporation per culture, approximately $1.5 \mathrm{mg}$ and $1 \times 10^{7}$ c.p.m., respectively).

and what we consider the most likely explanation for them.

The mutant $p f a-5$ most resembled wild-type in lipid composition and incorporation of exogenous acetate and $18: 1$. The reduced levels of polyunsaturates in this mutant are apparently not due to impaired desaturase activity (see Fig. 3). In this mutant, both the amount and the incorporation of acetate-derived label in PE, PC and DG relative to TG were on average higher than for wild-type (Table 3). The higher incorporation into DG was significant. Additionally, the absolute amount of label in TG averaged $20 \%$ higher than in wild-type.

A mutation reducing the level of PC biosynthesis by the minor CDP-choline:DG choline phosphotransferase (Kennedy) pathway (Weete, 1980) would cause reduced levels of polyunsaturates, since utilization of this pathway can return 18:1 or 18:2 from DG to PC, the substrate for further desaturation. This defect could cause the observed effects, namely accumulation of label in DG, and a compensating increase in the flux of FA through the main route of PC synthesis, via methylation of PE. Furthermore, since the rate-limiting step in TG biosynthesis in some organisms is DG acyltransferase (Frentzen, 1993), an accumulation of DG could enhance TG biosynthesis. It is unclear why this mutant might require $18: 3$ supplementation. Higher 18:3 levels may be required for an unknown biochemical function, or supplementation may compensate structurally for the higher level of saturates incorporated into lipids of the mutant.

$p f a-3$, as noted above, incorporated an extremely high proportion of the total acetate- or 18:1-derived label into free FA. $p f a-3$ cultures also had eight times the wild-type level of free FA, relative to the amount of TG produced. The acetate-derived label in free FA was mostly found in 16:0 and 18:0, as for wild-type; however, the free FA bulk composition differed: in wild-type, $38 \%$ of the free FA was 16:0, and $10 \% 18: 3$, whereas in the mutant $p f a-$ $3,15 \%$ was $16: 0$, and $37 \% 18: 3$. The $18: 3$ in the free FA pool, since unlabelled, was probably from the supplement. These observations suggest that $p f a-3$ most likely has a defect that interferes with acylation, such as a defective acyl-CoA synthase.

The label incorporation profiles of mutant $p f a-4$ superficially resembled those of $p f a-3$, but $p f a-4$ was distinctive in several respects. This mutant was capable of desaturation of $18: 1$ to $18: 2$ (see above), but had difficulty in further desaturating 18:2, and in its transfer to DG and TG: $\left[{ }^{14} \mathrm{C}\right]$ acetate-derived 18:2 was higher than for wildtype in PC (Fig. 2), but lower than wild-type in DG (not shown) and TG (Fig. 1a). The levels of the exogenously added 18:3 in TG (Fig. 1b) and PC (not shown) were also significantly low for this mutant. Other unusual characteristics of the mutant are that $\left[{ }^{14} \mathrm{C}\right]$ acetate-derived label was high (1.8 times wild-type) in PC relative to TG, and the amount of PC and DG also averaged comparatively more than for wild-type (Table 3). Also, as noted above, supplementation with high levels of exogenous $18: 1$ in the form of Tween 80 restored near wild-type growth. We suspect that this mutant either has a defect in the incorporation of unsaturated FA into complex lipids, most likely at the second acylation step, or that it has a mutation affecting electron transport which affects the second desaturation step to a greater degree. Mutations in 
the NADPH biosynthetic pathway reduce 18:3 levels in lipids of Neurospora (Brody \& Nyc, 1970). Either of these alternatives might be corrected by culture supplementation with high levels of unsaturated FA.

The mutants $p f a-1$ and $p f a-2$ both appear to be impaired in the desaturation of $18: 2$ to $18: 3$. The mutants differed in one respect, the incorporation of $\left[{ }^{14} \mathrm{C}\right]$ acetate-derived label into DG. In $p f a-1$, the label was primarily in the saturates (16:0 had an unusually high percentage of the total label), with some label detectable in $18: 2$. In contrast, $p f a-2$ had an unusually low amount of $16: 0$, both compared to wild-type and compared to the levels in PC. The label in 18:2 of DG was higher than for wild-type. Apparently, the two mutants have different selectivity for those molecular species of PC (or an alternative precursor) which are converted to DG. We suspect that one of these two is a 18:2 desaturase mutant, but do not know which DG incorporation profile is more likely for such a mutant.

\section{Implications for the control of polyunsaturated FA biosynthesis in Neurospora}

Four of the mutants we have isolated made no $18: 3$ in the $15^{\circ} \mathrm{C}$ screening, but did make $18: 3$ at reduced levels at $23^{\circ} \mathrm{C}$ (compare Table 1 and Fig. 1a). Alternative desaturation or elongation products of $\alpha-18: 2$ were not detected in wild-type or mutant lipids. Isolation of a set of four temperature-sensitive mutations, or mutations leaky at high temperature, is possible given the selection protocol. The results could alternatively be attributable to a second 18:2 desaturase or desaturation pathway in Neurospora. The substrate for desaturation to $\alpha-18: 3$ is believed to be 18:2-PC, but studies of 18:2 desaturation in Neurospora have been rare (Weete, 1980), and most have examined effects of temperature (Martin et al., 1981; Aaronson et al., 1982; Martin \& Johnston, 1983). It is not known which acyl group(s) undergoes the desaturation, and alternative substrates, such as CoA esters, which might be utilized in a minor pathway, have not been ruled out (Baker \& Lynen, 1971). Since NADPH biosynthetic mutants have reduced $18: 3$ levels (20\% of wild-type), it has been postulated that the 18:2 desaturase may utilize NADPH preferentially for the desaturation (Brody \& Nyc, 1970), whereas the 18:1 desaturase utilizes NADH (Gabrielides et al., 1982). A small amount of the total 18:3 may thus be formed by a second desaturase or using an alternative reductase. Most likely, given the absence of $18: 3$ synthesis by the mutants at $15^{\circ} \mathrm{C}$, only one desaturation system is utilized at this temperature. The preferential use of an 18:1-CoA desaturase over an 18:1PC desaturase at low temperature has been reported for the fungus Candida lipolytica (Pugh \& Kates, 1975). Evidence for an alternative desaturation pathway in $R$ hodotorula gracilis that is resistant to sterculate inhibition has also been presented (Rolph et al., 1990).

Our study has found that several mutations in Neurospora reduced the synthesis of polyunsaturated $F A$, indicating that multiple genes influence the polyunsaturated FA composition of membrane and storage lipids. Each of these mutations also showed genetic instability. The strong selection pressure against the mutants may be due to the specific biochemical defects or to the resulting effect on FA composition. Supplementation of the medium with 18:3 allowed the mutants to grow, and produced fertile crosses, suggesting that at least part of the phenotype resulted from failure to produce adequate levels of $18: 3$. However, even with supplementation, the mutants grew more slowly than wild-type. It is possible that some of the differences in precursor incorporation into the polyunsaturates result from the slower growth rate. Most notably, reduced levels of both $18: 2$ and $18: 3$ were synthesized de novo by each of the mutants at $23{ }^{\circ} \mathrm{C}$, even those which, judged by the criterion of ability to further desaturate $\left[{ }^{14} \mathrm{C}\right]$ oleate, were selectively impaired in the synthesis of 18:3. This effect contrasts with observations made in studies of Neurospora phospholipid composition during temperature changes, in which the amount of 18:2 and 18:3 appear to be controlled independently (Martin et al., 1981). However, the effect resembles one observed for ufa mutants, which do not desaturate 18:0, but may also show impaired 18:1 or 18:2 desaturation (Scott, 1977b; Gabrielides et al., 1982; Goodrich-Tanrikulu et al., 1994).

More definitive characterization of the defects in these mutants requires additional study. Unfortunately, only limited information is available on the relevant biochemical pathways in Neurospora (Weete, 1980; Chopra \& Khuller, 1984). Mutants in the FA desaturation pathway of Arabidopsis have been extremely valuable for identifying the control of the corresponding plant pathway, for observing physiological effects of altered FA composition, and for isolation of the genes involved (reviewed by Heinz, 1993; Gibson et al., 1994). Likewise, further studies in Neurospora will identify similarities and differences in FA biosynthesis of filamentous fungi, and should provide insight into related processes in higher plants.

\section{ACKNOWLEDGEMENTS}

We thank Benjamin E. Gordon at Lawrence Berkeley Laboratories for performing irradiation of conidia, David D. Perkins at Stanford University for advice on crosses, and Stuart Brody (University of California, San Diego) for several discussions on fatty acid biosynthetic mutants.

\section{REFERENCES}

Aaronson, L. R., Johnston, A. M. \& Martin, C. E. (1982). The effects of temperature acclimation on membrane sterols and phospholipids of Neurospora crassa. Biocbim Biophys Acta 713, 456-462.

Baker, N. \& Lynen, F. (1971). Factors involved in fatty acyl CoA desaturation by fungal microsomes. The relative roles of acyl CoA and phospholipids as substrates. Eur J Biochem 19, 200-210.

Brody, S. \& Nyc, J.F. (1970). Altered fatty acid distribution in mutants of Neurospora crassa. J Bacteriol 104, 780-786.

Chopra, A. \& Khuller, G. K. (1984). Lipid metabolism in fungi. CRC Crit Rev Microbiol 11, 209-271.

Coté, G. G. \& Brody, S. (1987). Circadian rhythms in Neurospora crassa : membrane composition of a mutant defective in temperature compensation. Biochim Biophys Acta 898, 23-36. 
Davis, R. H. \& deSerres, F. J. (1970). Genetic and microbiological research techniques for Neurospora crassa. Methods Enzymol 17A, 79-143.

Frentzen, M. (1993). Acyltransferases and triacylglycerols. In Lipid Metabolism in Plants, pp. 195-230. Edited by T. S. Moore, Jr. Boca Raton: CRC Press.

Gabrielides, C., Hamill, A. L. \& Scott, W. A. (1982). Requirements of $\Delta^{9}$ and $\Delta^{12}$ fatty acid desaturation in Neurospora. Biochim Biophys Acta 712, 505-514.

Gibson, S., Falcone, D. L., Browse, J. \& Somerville, C. (1994). Use of transgenic plants and mutants to study the regulation and function of lipid composition. Plant Cell Environ 17, 627-637.

Goodrich-Tanrikulu, M., Stafford, A. E., Lin, J.-T., Makapugay, M. I., Fuller, G. \& McKeon, T. A. (1994). Fatty acid biosynthesis in novel ufa mutants of Neurospora crassa. Microbiology 140, 2683-2690.

Heinz, E. (1993). Biosynthesis of polyunsaturated fatty acids. In Lipid Metabolism in Plants, pp. 33-89. Edited by T. S. Moore, Jr. Boca Raton: CRC Press.

Horowitz, N. H. \& Beadle, G. W. (1943). A microbiological method for the determination of choline by use of a mutant of Neurospora. J Biol Chem 159, 145-151.

Horowitz, N. H., Bonner, D. \& Houlahan, M. B. (1945). The utilization of choline analogues by cholineless mutants of Neurospora. J Biol Chem 150, 325-333.

Hubbard, S. C. \& Brody, S. (1975). Glycerophospholipid variation in choline and inositol auxotrophs of Neurospora crassa. J Biol Cbem 250, 7173-7181.

Jareonkitmongkol, S., Shimizu, S. \& Yamada, H. (1992). Fatty acid desaturation-defective mutants of an arachidonic-acid-producing fungus, Mortierella alpina 1S-4. J Gen Microbiol 138, 997-1002.

Johnston, A. M., Aaronson, L. R. \& Martin, C. E. (1982). The effects of altered levels of phosphatidylcholine and phosphatidylethanolamine on fatty acid desaturase activity and sterol metabolism during temperature acclimation in a choline auxotroph of Neurospora crassa. Biocbim Biopbys Acta 713, 512-518.

Martin, C. E. \& Johnston, A. M. (1983). Changes in fatty acid distribution and thermotropic properties of phospholipids following phosphatidylcholine depletion in a choline-requiring mutant of Neurospora crassa. Biochim Biophys Acta 730, 10-16.
Martin, C. E., Siegel, D. \& Aaronson, L. R. (1981). Effects of temperature acclimation on Neurospora phospholipids: fatty acid desaturation appears to be a key element in modifying phospholipid fluid properties. Biocbim Biopbys Acta 665, 399-407.

Mitchell, M. B. \& Mitchell, H. K. (1950). The selective advantage of an adenineless double mutant over one of the single mutants involved. Proc Natl Acad Sci USA 36, 115-119.

Murray, N. E. (1965). Cysteine mutant strains of Neurospora crassa. Genetics 52, 1163-1183.

Nyc, J. F. \& Brody, S. (1971). Effects of mutations and growth conditions on lipid synthesis in Neurospora crassa. J Bacteriol 108, 1310-1317.

Perkins, D. D. (1994). Deviations from 1:1 and numbers of progeny necessary for establishing linkage. Fungal Genet Newsl 41, 69-70.

Pugh, E. L. \& Kates, M. (1975). Characterization of a membranebound phospholipid desaturase system of Candida lipolytica. Biocbim Biopbys Acta 380, 442-453.

Rolph, C. E., Moreton, R. S. \& Harwood, J. L. (1990). Control of acyl lipid desaturation in the yeast $\mathrm{R}$ hodotorula gracilis via the use of the cyclopropenoid fatty acid, sterculate. Appl Microbiol Biotechnol 34, 91-96.

Scarborough, G. A. \& Nyc, J. F. (1967). Methylation of ethanolamine phosphatides by microsomes from normal and mutant strains of Neurospora crassa. J Biol Chem 242, 238-242.

Scott, W. A. (1977a). Unsaturated fatty acid mutants of Neurospora crassa. J Bacteriol 130, 1144-1148.

Scott, W. A. (1977b). Mutations resulting in an unsaturated fatty acid requirement in Neurospora. Evidence for $\Delta^{9}$-desaturase defects. Biochemistry 16, 5274-5279.

Wada, H. \& Murata, N. (1989). Synechocystis PCC6803 mutants defective in desaturation of fatty acids. Plant Cell Physiol 30, 971-978.

Weete, J. D. (1980). Lipid Biochemistry of Fungi and Other Organisms. New York: Plenum Press.

Received 23 December 1994; revised 7 April 1995; accepted 21 April 1995. 